Journal of Applied AnALysis

Vol. 9, No. 2 (2003), pp. 287-299

\title{
NEW AND GENERALIZED CONVERGENCE CONDITIONS FOR THE NEWTON-KANTOROVICH METHOD
}

\author{
I. K. ARGYROS \\ Received August 8, 2002 and, in revised form, June 23, 2003
}

\begin{abstract}
We present new semilocal convergence theorems for Newton methods in a Banach space. Using earlier general conditions we find more precise error estimates on the distances involved using the majorant principle. Moreover we provide a better information on the location of the solution. In the special case of Newton's method under Lipschitz conditions we show that the famous Newton-Kantorovich hypothesis having gone unchallenged for a long time can be weakened under the same hypotheses/computational cost.
\end{abstract}

\section{Introduction}

In this study we are concerned with the problem of approximating a locally unique solution $x^{*}$ of equation

$$
F(x)=0,
$$

2000 Mathematics Subject Classification. 65H10, 47H17, 49M15.

Key words and phrases. Newton's method, Banach space, majorant method, NewtonKantorovich theorem/hypothesis, Fréchet-derivative.

ISSN 1425-6908 (C) Heldermann Verlag. 
where, $F$ is a Fréchet-differentiable operator defined on a closed ball $\bar{U}\left(x_{0}, R\right)=\left\{x \in X \mid\left\|x-x_{0}\right\| \leq R\right\}(R>0)$ which is a subset of a Banach space $X$ with values in a Banach space $Y$.

A large number of problems in applied mathematics and also in engineering are solved by finding the solutions of certain equations. For example, dynamic systems are mathematically modeled by difference or differential equations, and their solutions usually represent the states of the systems. For the sake of simplicity, assume that a time-invariant system is driven by the equation $\dot{x}=F(x)$, where $x$ is the state. Then the equilibrium states are determined by solving equation (1). Similar equations are used in the case of discrete systems. The unknowns of engineering equations can be functions (difference, differential, and integral equations), vectors (systems of linear or nonlinear algebraic equations), or real or complex numbers (single algebraic equations with single unknowns). Except in special cases, the most commonly used solution methods are iterative - when starting from one or several initial approximations a sequence is constructed that converges to a solution of the equation. Iteration methods are also applied for solving optimization problems. In such cases, the iteration sequences converge to an optimal solution of the problem at hand. Since all of these methods have the same recursive structure, they can be introduced and discussed in a general framework.

Newton's method

$$
x_{n+1}=x_{n}-F^{\prime}\left(x_{n}\right)^{-1} F\left(x_{n}\right) \quad(n \geq 0)
$$

has been used extensively and under various conditions to generate a sequence approximating $x^{*}$. A survey of local and semilocal convergence theorems for Newton's method under various conditions on the Fréchetderivative $F^{\prime}(x)$ of operator $F(x)$ can be found in [3], [4], [7], [9], [11].

Recently De Pascale and Zabrejko in [5] (see the references there also) gave some semilocal convergence results using very general conditions. In particular, they provided semilocal results by replacing the usual Lipschitz continuity conditions by a "small" majorizing monotonically increasing function (see (7)).

Here under very similar conditions, via the majorant principle we introduce more precise error estimates on the distances $\left\|x_{n+1}-x_{n}\right\|,\left\|x_{n}-x^{*}\right\|$ $(n \geq 0)$. Moreover we provide a better information on the location of the solution $x^{*}$. Finally we show as an application/special case that the famous Newton-Kantorovich hypothesis (see (53)) [7] essentially having been used unchallenged by Cauchy, Fourier and many others ever since can be weakened (see (50)) under the same hypotheses/computational cost. This observation is very important in computational mathematics since it allows 
a wider choice of initial guesses $x_{0}$ and finer error bound on the distances involved [1]-[4], [6], [8], [10].

\section{Semilocal analysis of Newton's method}

Given functions $w_{0}, w$ defined on $[0, \infty)$ with values in $[0, \infty)$, and parameter $R>0$ it is convenient for the presentation of Theorem 1 to define functions $\bar{w}, \bar{\varphi}$ on $[0, R]$ by

$$
\begin{aligned}
& \bar{w}(r)=\sup \left\{w\left(v_{1}\right)+w_{0}\left(v_{2}\right): v_{1}+v_{2}=r\right\}, \\
& \bar{\varphi}(r)=\eta-r+\int_{0}^{r} \bar{w}(t) d t
\end{aligned}
$$

and iteration $\left\{r_{n}\right\}(n \geq 0)$ by

$$
r_{0}=0, \quad r_{n+1}=r_{n}+\frac{\bar{\varphi}\left(r_{n}\right)}{1-w_{0}\left(r_{n}\right)} \quad(n \geq 0) .
$$

We state the following semilocal convergence result from [5] for Newton's method:

Theorem 1. Let $F: \bar{U}\left(x_{0}, R\right) \subseteq X \rightarrow Y$ be a Fréchet-differentiable operator. Assume there exist $R>0$, and $x_{0} \in X$ with $F^{\prime}\left(x_{0}\right)^{-1} \in L(Y, X)$ the space of bounded linear operators from $Y$ into $X$, such that:

$$
\begin{aligned}
\left\|F^{\prime}\left(x_{0}\right)^{-1} F\left(x_{0}\right)\right\| & \leq \eta \\
\left\|F^{\prime}\left(x_{0}\right)^{-1}\left[F^{\prime}(x)-F^{\prime}(y)\right]\right\| & \leq w(\|x-y\|), \quad\left(x, y \in \bar{U}\left(x_{0}, R\right)\right)
\end{aligned}
$$

where $\eta \in[0, R]$, and function $w$ is monotonically increasing with

$$
\lim _{r \rightarrow 0} w(r)=0 .
$$

Moreover, assume function $w_{0}$ is monotonically increasing such that

$$
0 \leq w_{0}(r) \leq w(r) \quad(0 \leq r \leq R)
$$

and

$$
\left\|F^{\prime}(x)^{-1} F^{\prime}\left(x_{0}\right)\right\| \leq\left(1-w_{0}(r)\right)^{-1} \quad\left(x \in \bar{U}\left(x_{0}, r\right)\right) .
$$

Furthermore, function $\bar{\varphi}$ has a unique zero $r^{*} \in[0, R]$ and

$$
\bar{\varphi}(R) \leq 0 \text {. }
$$

Then,

(i) scalar sequence $\left\{r_{n}\right\}(n \geq 0)$ converges monotonically to $r^{*}$. 
(ii) sequence $\left\{x_{n}\right\}(n \geq 0)$ generated by Newton's method (2) is well defined, remains in $\bar{U}\left(x_{0}, r^{*}\right)$ for all $n \geq 0$ and converges to a solution $x^{*} \in \bar{U}\left(x_{0}, r^{*}\right)$ of equation (1). Moreover the following error bounds hold for all $n \geq 0$,

$$
\left\|x_{n+1}-x_{n}\right\| \leq r_{n+1}-r_{n}
$$

and

$$
\left\|x_{n}-x^{*}\right\| \leq r^{*}-r_{n}
$$

Note that under the hypotheses of Theorem 1 it was shown in [5] that $w_{0}\left(r_{n}\right) \neq 1$ for all $n \geq 0$ and $1-w_{0}(r)>0$ for all $r \in[0, R]$.

We will need the following result on the convergence of majorizing sequences.

Theorem 2. Assume there exist $\delta \in[0,2)$, parameter $\eta$, functions $w, w_{0}$ as in (6), (7), and (9) respectively, such that:

$$
\begin{gathered}
h_{\delta}=2 \int_{0}^{1} w(\theta \eta) d \theta+\delta w_{0}(\eta) \leq \delta, \\
w_{0}\left[\frac{2 \eta}{2-\delta}\left(1-\left(\frac{\delta}{2}\right)^{n+1}\right)\right]<1,
\end{gathered}
$$

and

$$
\begin{aligned}
& \delta\left\{w_{0}\left[\frac{2 \eta}{2-\delta}\left(1-\left(\frac{\delta}{2}\right)^{n+2}\right)\right]-w_{0}(\eta)\right\} \\
& \leq 2 \int_{0}^{1}\left[w(\theta \eta)-w\left(\theta\left(\frac{\delta}{2}\right)^{n+1} \eta\right)\right] d \theta \quad(n \geq 0) .
\end{aligned}
$$

Then, iteration $\left\{t_{n}\right\}(n \geq 0)$ given by

$$
\begin{aligned}
& t_{0}=0, t_{1}=\eta, \\
& 0 \leq t_{n+2}=t_{n+1}+\frac{\int_{0}^{1} w\left[\theta\left(t_{n+1}-t_{n}\right)\right] d \theta}{1-w_{0}\left(t_{n+1}\right)}\left(t_{n+1}-t_{n}\right)
\end{aligned}
$$

is non-decreasing, bounded above by

$$
t^{* *}=\frac{2 \eta}{2-\delta},
$$

and converges to some $t^{*}$ such that

$$
0 \leq t^{*} \leq t^{* *} .
$$


Moreover, the following error bounds hold for all $n \geq 0$

$$
0 \leq t_{n+2}-t_{n+1} \leq \frac{\delta}{2}\left(t_{n+1}-t_{n}\right) \leq\left(\frac{\delta}{2}\right)^{n+1} \eta
$$

Proof. We must show:

$$
2 \int_{0}^{1} w\left(\theta\left(t_{k+1}-t_{k}\right)\right) d \theta+\delta w_{0}\left(t_{k+1}\right) \leq \delta
$$

and

$$
1-w_{0}\left(t_{k+1}\right)>0
$$

for all $k \geq 0$.

Estimate (20) can then follow immediately from (21) and (22). Using induction on the integer $k$ we have for $k=0$

$$
2 \int_{0}^{1} w\left[\theta\left(t_{1}-t_{0}\right)\right] d \theta+\delta w_{0}\left(t_{1}\right)=2 \int_{0}^{1} w(\theta \eta) d \theta+\delta w_{0}(\eta) \leq \delta
$$

and

$$
1-w_{0}\left(t_{1}\right)=1-w_{0}(\eta)>0 \quad(\text { by }(15)) \text {. }
$$

But (17) gives

$$
0 \leq t_{2}-t_{1} \leq \frac{\delta}{2}\left(t_{1}-t_{0}\right)
$$

Assume (21) and (22) hold for all $k \leq n+1$. We obtain in turn

$$
\begin{aligned}
& 2 \int_{0}^{1} w\left[\theta\left(t_{k+2}-t_{k+1}\right)\right] d \theta+\delta w_{0}\left(t_{k+2}\right) \\
& \leq 2 \int_{0}^{1} w\left[\theta\left(\frac{\delta}{2}\right)^{k+1} \eta\right] d \theta+\delta w_{0}\left[\frac{2 \eta}{2-\delta}\left(1-\left(\frac{\delta}{2}\right)^{k+2}\right)\right] \\
& \leq 2 \int_{0}^{1} w(\theta \eta) d \theta+\delta w_{0}(\eta) \leq \delta \quad(\text { by }(14) \text { and }(16))
\end{aligned}
$$

Hence, estimate (20) holds for all $k \geq 0$. Moreover, we must show:

$$
t_{k} \leq t^{* *}
$$

We have

$$
t_{0}=0 \leq t^{* *}, \quad t_{1}=\eta \leq t^{* *} \quad \text { and } \quad t_{2} \leq \eta+\frac{\delta}{2} \eta=\frac{2+\delta}{2} \eta \leq t^{* *} .
$$


Assume (24) holds for all $k \leq n+1$. It follows from (20)

$$
\begin{aligned}
t_{k+2} & \leq t_{k+1}+\frac{\delta}{2}\left(t_{k+1}-t_{k}\right) \leq t_{k}+\frac{\delta}{2}\left(t_{k}-t_{k-1}\right)+\frac{\delta}{2}\left(t_{k+1}-t_{k}\right) \\
& \leq \cdots \leq \eta+\frac{\delta}{2} \eta+\left(\frac{\delta}{2}\right)^{2} \eta+\cdots+\left(\frac{\delta}{2}\right)^{k+1} \eta \\
& \leq \frac{1-\left(\frac{\delta}{2}\right)^{k+2}}{1-\frac{\delta}{2}} \leq \frac{2}{2-\delta} \eta=t^{* *} .
\end{aligned}
$$

Hence, sequence $\left\{t_{n}\right\}(n \geq 0)$ is bounded above by $t^{* *}$.

Moreover (22) follows from (15) and the above.

Furthermore, sequence $\left\{t_{n}\right\}(n \geq 0)$ is non-decreasing by (17) and as such it converges to some $t^{*}$ satisfying (19).

That completes the proof of Theorem 2 .

Below we present the main result on the semilocal convergence of Newton's method. For relevant results, see also [1], [2], [6], [8].

Theorem 3. Suppose that the hypotheses of Theorem 2,

$$
\left\|F^{\prime}\left(x_{0}\right)^{-1}\left[F^{\prime}(x)-F^{\prime}\left(x_{0}\right)\right]\right\| \leq w_{0}\left(\left\|x-x_{0}\right\|\right) \quad x \in \bar{U}\left(x_{0}, R\right),
$$

and

$$
t^{*} \leq R,
$$

hold.

Then, sequence $\left\{x_{n}\right\}(n \geq 0)$ generated by Newton's method (2) is well defined, remains in $\bar{U}\left(x_{0}, t^{*}\right)$ for all $n \geq 0$ and converges to a solution $x^{*} \in \bar{U}\left(x_{0}, t^{*}\right)$ of equation $F(x)=0$.

Moreover, the following error bounds hold for all $n \geq 0$

$$
\left\|x_{n+1}-x_{n}\right\| \leq t_{n+1}-t_{n}
$$

and

$$
\left\|x_{n}-x^{*}\right\| \leq t^{*}-t_{n},
$$

where, iteration $\left\{t_{n}\right\}(n \geq 0)$ is given by (17). Furthermore, if there exists $R_{0}>t^{*}$ such that:

$$
\begin{gathered}
R_{0} \leq R, \\
\int_{0}^{1} w_{0}\left[\left(\theta t^{*}+(1-\theta) R_{0}\right) d \theta\right] \leq 1,
\end{gathered}
$$

then the solution $x^{*}$ is unique in $U\left(x_{0}, R_{0}\right)$, and if

$$
w_{0}\left(t^{*}\right)<1 \text {, }
$$


the solution is unique in $\bar{U}\left(x_{0}, t^{*}\right)$.

Proof. We must show estimate (27). For $n=0,(27)$ is obvious, since

$$
\left\|x_{1}-x_{0}\right\|=\left\|F^{\prime}\left(x_{0}\right)^{-1} F\left(x_{0}\right)\right\| \leq \eta=t_{1}-t_{0} \quad(\text { by }(6)) .
$$

Suppose (27) holds for $n=0,1, \ldots, k+1$; this implies, in particular that

$$
\begin{aligned}
\left\|x_{k+1}-x_{0}\right\| & \leq\left\|x_{k+1}-x_{k}\right\|+\left\|x_{k}-x_{k-1}\right\|+\cdots+\left\|x_{1}-x_{0}\right\| \\
& \leq\left(t_{k+1}-t_{k}\right)+\left(t_{k}-t_{k-1}\right)+\cdots+\left(t_{1}-t_{0}\right) \\
& =t_{k+1}-t_{0}=t_{k+1} .
\end{aligned}
$$

We show that (27) holds for $n=k+2$. Using (25) and (15) we get

$$
\begin{aligned}
\left\|F^{\prime}\left(x_{0}\right)^{-1}\left(F^{\prime}\left(x_{k+1}\right)-F^{\prime}\left(x_{0}\right)\right)\right\| & \leq w_{0}\left(\left\|x_{k+1}-x_{0}\right\|\right) \\
& \leq w_{0}\left(t_{k+1}\right)<1
\end{aligned}
$$

It follows from (32) and the Banach Lemma on invertible operators [7] that $F^{\prime}\left(x_{k+1}\right)^{-1}$ exists and

$$
\left\|F^{\prime}\left(x_{k+1}\right)^{-1} F^{\prime}\left(x_{0}\right)\right\| \leq \frac{1}{1-w_{0}\left(\left\|x_{k+1}-x_{0}\right\|\right)} \leq \frac{1}{1-w_{0}\left(t_{k+1}\right)} .
$$

By (7) we obtain

$$
\begin{aligned}
& \left\|F^{\prime}\left(x_{0}\right)^{-1} F\left(x_{k+1}\right)\right\| \\
& =\left\|F^{\prime}\left(x_{0}\right)^{-1} \int_{0}^{1}\left[F^{\prime}\left((1-\theta) x_{k}+\theta x_{k+1}\right)-F^{\prime}\left(x_{k}\right)\right]\left(x_{k+1}-x_{k}\right) d \theta\right\| \\
& \leq \int_{0}^{1}\left\|F^{\prime}\left(x_{0}\right)^{-1}\left[F^{\prime}\left((1-\theta) x_{k}+\theta x_{k+1}\right)-F^{\prime}\left(x_{k}\right)\right]\right\|\left\|x_{k+1}-x_{k}\right\| d \theta \\
& \leq \int_{0}^{1} w\left[\theta\left(t_{k+1}-t_{k}\right)\right]\left(t_{k+1}-t_{k}\right) d \theta
\end{aligned}
$$

Hence, by (2), (33) and (34) we get

$$
\begin{aligned}
\left\|x_{k+2}-x_{k+1}\right\| & =\left\|F^{\prime}\left(x_{k+1}\right)^{-1} F\left(x_{k+1}\right)\right\| \\
& \leq\left\|F^{\prime}\left(x_{k+1}\right)^{-1} F^{\prime}\left(x_{0}\right)\right\| \cdot\left\|F^{\prime}\left(x_{0}\right)^{-1} F\left(x_{k+1}\right)\right\| \\
& \leq \frac{\int_{0}^{1} w\left[\theta\left(t_{k+1}-t_{k}\right)\right]\left(t_{k+1}-t_{k}\right) d \theta}{1-w_{0}\left(t_{k+1}\right)}=t_{k+2}-t_{k+1}
\end{aligned}
$$

which shows (27) for all $n \geq 0$.

It follows that $\left\{x_{n}\right\}(n \geq 0)$ is a Cauchy sequence in a Banach space $X$ and such it converges to some $x^{*} \in \bar{U}\left(x_{0}, t^{*}\right)$ (since $\bar{U}\left(x_{0}, t^{*}\right)$ is a closed set). By letting $k \rightarrow \infty$ in (35) we obtain $F\left(x^{*}\right)=0$. Moreover, estimate (28) follows from (27) by using standard majorization techniques. Furthermore, 
to show uniqueness, let $y^{*}$ be a solution of equation $F(x)=0$ in $U\left(x_{0}, R_{0}\right)$. It follows from the estimate

$$
\begin{aligned}
& \left\|F^{\prime}\left(x_{0}\right)^{-1} \int_{0}^{1}\left[F^{\prime}\left(y^{*}+\theta\left(x^{*}-y^{*}\right)\right)-F^{\prime}\left(x_{0}\right)\right] d \theta\right\| \\
& \leq \int_{0}^{1} w_{0}\left\|y^{*}+\theta\left(x^{*}-y^{*}\right)-x_{0}\right\| d \theta \\
& \leq \int_{0}^{1} w_{0}\left[\theta\left\|x^{*}-x_{0}\right\|+(1-\theta)\left\|y^{*}-x_{0}\right\|\right] d \theta \\
& <\int_{0}^{1} w_{0}\left[\theta t^{*}+(1-\theta) R_{0}\right] d \theta \leq 1, \quad(\text { by }(30))
\end{aligned}
$$

and the Banach lemma on invertible operators that linear operator

$$
L=\int_{0}^{1} F^{\prime}\left[y^{*}+\theta\left(x^{*}-y^{*}\right)\right] d \theta
$$

is invertible.

Using the identity

$$
0=F\left(x^{*}\right)-F\left(y^{*}\right)=L\left(x^{*}-y^{*}\right),
$$

we deduce

$$
x^{*}=y^{*} .
$$

The second result on the uniqueness follows similarly but using (31).

That completes the proof of Theorem 3 .

In the next result we show: our error bounds (27), (28) are more precise than (12), (13) respectively. We also provide a better information on the location of the solution $x^{*}$.

Theorem 4. Under the hypotheses of Theorems 1 and 3 the following hold for all $n \geq 0$ :

$$
\begin{aligned}
t_{0} & =r_{0}=0, \quad t_{1}=\eta \leq r_{1}=\frac{\eta}{1-w_{0}(0)}, \\
t_{n+1} & \leq r_{n+1} \\
t_{n+1}-t_{n} & \leq r_{n+1}-r_{n} \\
t^{*}-t_{n} & \leq r^{*}-r_{n}
\end{aligned}
$$

and

$$
t^{*} \leq r^{*} .
$$


Proof. We use induction on the integer $k$ to show (37) and (38). For $n=0$ in (17) we obtain

$$
\begin{aligned}
t_{2}-t_{1} & =\frac{\int_{0}^{1} w\left[\theta\left(t_{1}-t_{0}\right)\right]\left(t_{1}-t_{0}\right) d \theta}{1-w_{0}\left(t_{1}\right)} \\
& =\frac{\int_{0}^{1} w\left[r_{0}+\theta\left(r_{1}-r_{0}\right)-r_{0}\right]\left(r_{1}-r_{0}\right) d \theta}{1-w_{0}\left(r_{1}\right)} \\
& \leq \frac{\int_{0}^{1}\left\{\bar{w}\left[r_{0}+\theta\left(r_{1}-r_{0}\right)\right]-w_{0}\left(r_{0}\right)\right\}\left(r_{1}-r_{0}\right) d \theta}{1-w_{0}\left(r_{1}\right)} \\
& \leq \frac{\bar{\varphi}\left(r_{1}\right)-\bar{\varphi}\left(r_{0}\right)-\left(w_{0}\left(r_{0}\right)-1\right)\left(r_{1}-r_{0}\right)}{1-w_{0}\left(r_{1}\right)} \\
& =\frac{\bar{\varphi}\left(r_{1}\right)}{1-w_{0}\left(r_{1}\right)}=r_{2}-r_{1},
\end{aligned}
$$

and

$$
t_{2} \leq r_{2}
$$

Assume:

$$
t_{k+1} \leq r_{k+1}
$$

and

$$
t_{k+1}-t_{k} \leq r_{k+1}-r_{k}
$$

for all $k \leq n$.

Using (17), (41) and (42) we get in turn:

$$
\begin{aligned}
t_{k+2}-t_{k+1} & =\frac{\int_{0}^{1} w\left[\theta\left(t_{k+1}-t_{k}\right)\right]\left(t_{k+1}-t_{k}\right) d \theta}{1-w_{0}\left(t_{k+1}\right)} \\
& \leq \frac{\int_{0}^{1} w\left[r_{k}+\theta\left(r_{k+1}-r_{k}\right)-r_{k}\right]\left(r_{k+1}-r_{k}\right) d \theta}{1-w_{0}\left(r_{k+1}\right)} \\
& \leq \frac{\int_{0}^{1}\left\{\bar{w}\left[r_{k}+\theta\left(r_{k+1}-r_{k}\right)\right]-w_{0}\left(r_{k}\right)\right\}\left(r_{k+1}-r_{k}\right) d \theta}{1-w_{0}\left(r_{k+1}\right)} \\
& \leq \frac{\bar{\varphi}\left(r_{k+1}\right)-\bar{\varphi}\left(r_{k}\right)-\left[w_{0}\left(r_{k}\right)-1\right]\left(r_{k+1}-r_{k}\right)}{1-w_{0}\left(r_{k+1}\right)} \\
& =\frac{\bar{\varphi}\left(r_{k+1}\right)}{1-w_{0}\left(r_{k+1}\right)}=r_{k+2}-r_{k+1}
\end{aligned}
$$

and

$$
t_{k+2} \leq r_{k+2}
$$

which shows (37), (38) for all $n \geq 0$.

Let $m \geq 0$, then as above we can have:

$$
t_{k+m}-t_{k} \leq r_{k+m}-r_{k} .
$$


By letting $m \rightarrow \infty$ in (43) we obtain (39). Finally, set $n=0$ in (39) to obtain (40).

That completes the proof of Theorem 4 .

Remark 1. Hypotheses of the form (14), (30) and (31) are as easy to handle as (11) (see also (49) and (50)) and are always present as sufficient convergence conditions in the study of Newton's method (2) [3], [7]. Note that $t^{*}$ can be replaced by $2 \eta /(2-\delta)$ in condition $(26)$.

Remark 2. The assumptions (8) and (9) imply $w_{0}(0)=0$, then we obtain $t_{1}=r_{1}$. But if $t_{2}<r_{2}$ then again (37) and (38) hold as strict inequalities.

Remark 3. It can easily be seen from (21) that condition (16) can be dropped if (14) is replaced by

$$
2 \int_{0}^{1} w(\theta \eta) d \theta+\delta w_{0}\left(\frac{2 \eta}{2-\delta}\right) \leq \delta .
$$

Similarly condition $(15)$ can be replaced by $w_{0}[2 \eta /(2-\delta)] \leq 1$.

Remark 4. Condition (16) holds in many cases. For example, let

$$
w(r)=\ell r
$$

and

$$
w_{0}(r)=\ell_{0} r
$$

for some non-negative parameters $\ell, \ell_{0}$ with $\ell_{0} \leq \ell$. That is the original Newton-Kantorovich case. With the above choices (16) becomes

$$
\delta \ell_{0}\left\{\frac{2}{2-\delta}\left(1-\left(\frac{\delta}{2}\right)^{k+2}\right)-1\right\} \leq \ell\left[1-\left(\frac{\delta}{2}\right)^{k+1}\right],
$$

or

$$
\left[\frac{\ell_{0} \delta^{2}}{2-\delta}-\ell\right]\left[1-\left(\frac{\delta}{2}\right)^{k+1}\right] \leq 0
$$

or

$$
\frac{\ell_{0} \delta^{2}}{2-\delta} \leq \ell
$$

Moreover (15) holds if

$$
\frac{2 \ell_{0} \eta}{2-\delta} \leq 1
$$

Furthermore (14) holds if

$$
h_{\delta}=\left(\delta \ell_{0}+\ell\right) \eta \leq \delta .
$$


It can easily be seen that (47), (48) always hold if $\delta \in[0,1]$. That is in this case conditions (47)-(49) reduce to (49).

For the case of Theorem 2, set $\delta=1$ in (14) to obtain in particular:

$$
h_{1}=\left(\ell+\ell_{0}\right) \eta \leq 1 \text {. }
$$

Using the same choices of functions $w$ and $w_{0}$, let us also consider the case of Theorem 1. It can easily be seen from (3) that

$$
\bar{w}(r)=\ell r .
$$

Hence, $\bar{\varphi}$ has a zero $s^{*}$ given by

$$
r^{*}=\frac{1-\sqrt{1-h}}{\ell}
$$

provided that

$$
h=2 \ell \eta \leq 1 .
$$

Condition (53) is the famous Newton-Kantorovich hypothesis [7] essentially used since Newton's time as the crucial sufficient convergence condition for the convergence of Newton's method in this case.

We have

$$
h \leq 1 \Longrightarrow h_{1} \leq 1,
$$

but not vice versa unless if $\ell_{0}=\ell$. Hence the long standing hypothesis (53) has been weakened using the same information/computation cost. This observation is very important in computational mathematics and makes the choice of the initial guess $x_{0}$ much easier.

Note also:

$$
\ell_{0} \leq \ell
$$

in general. In case $\ell_{0}<\ell$, then $t_{2}<r_{2}$ and (37), (38) hold as strict inequalities (see also Remark 2 ). Hence we obtain more precise error bounds in this case.

Moreover $\ell / \ell_{0}$ can be arbitrarily large.

In the examples that follow the choices of functions $w, w_{0}, \bar{w}$ given by (45), (46) and (51) are used.

Example 1. Let $X=Y=\mathbb{R}, x_{0}=0$ and define function $F$ on $\mathbb{R}$ by

$$
F(x)=c_{0}+c_{1} x+c_{2} \sin e^{c_{3} x},
$$

where $c_{i}, i=0,1,2,3$ are given parameters. It can easily be seen using (56) for $c_{3}$ large and $c_{2}$ sufficiently small $\ell / \ell_{0}$ can be arbitrarily large. That is (50) may hold but not (53). 
Example 2. Let $X=Y=\mathbb{R}, \bar{U}\left(x_{0}, R\right)=\bar{U}(\sqrt{2}, 1)$ and define function $F$ on $\bar{U}$ by

$$
F(x)=\frac{1}{6} x^{3}-\left(\frac{2^{3 / 2}}{6}+0.23\right) .
$$

It can easily be seen that

$$
\begin{aligned}
& \eta=0.23, \quad \ell=2.4142136, \quad \ell_{0}=1.914213562, \\
& h=1.1105383>1 \text { and } h_{1}=0.995538247<1 .
\end{aligned}
$$

That is there is no guarantee that Newton's method starting at $x_{0}$ converges to $x^{*}=1.614507018$ since (49) is violated. However since (50) holds our results guarantee $\lim _{n \rightarrow \infty} x_{n}=x^{*}=1.614507018$.

Example 3. Let $X=Y=\mathbb{R}, x_{0}=1$ and define function $F$ by

$$
F(x)=x^{3}-a, \quad \text { for all } a \in\left[0, \frac{1}{2}\right), \quad x \in[a, 2-a] .
$$

Using (6), (7), (25) and (58) we obtain

$$
\eta=\frac{1}{3}(1-a), \quad \ell=2(2-a) \quad \text { and } \quad \ell_{0}=3-a .
$$

The Newton-Kantorovich hypothesis (53) is violated since

$$
h=\frac{4}{3}(1-a)(2-a)>1 \quad \text { for all } a \in\left[0, \frac{1}{2}\right) .
$$

That is there is no guarantee that Newton's method (2) converges to the solution $x^{*}=\sqrt[3]{a}$ of equation $F(x)=0$. However our condition (50) guarantees convergence for all $a \in[(5-\sqrt{13}) / 3,1 / 2)$ since

$$
h_{1}=\frac{1}{3}(1-a)[3-a+2(2-a)] \leq 1 .
$$

\section{References}

[1] Argyros, I. K., Relation between forcing sequences and inexact Newton iterates in Banach space, Computing 63 (1999), 131-144.

[2] Argyros, I. K., Newton methods on Banach spaces with a convergence structure and applications, Comput. Math. Appl. 40(1) (2000), 37-48.

[3] Argyros, I. K., Advances in the Efficiency of Computational Methods and Applications, World Scientific Publ. Co., River Edge, NJ, 2000.

[4] Argyros, I. K., Szidarovszky, F., The Theory and Applications of Iteration Methods, CRC Press, Boca Raton, FL, 1993.

[5] De Pascale, E., Zabrejko, P. P., New convergence criterion for the Newton-Kantorovich method and some applications to nonlinear integral equations, Rend. Sem. Mat. Univ. Padova 100 (1998), 211-230. 
[6] Gutiérrez, J. M., A new semilocal convergence theorem for Newton's method, J. Comput. Appl. Math. 79 (1997), 131-145.

[7] Kantorovich, L. V., Akilov, G. P., Functional Analysis in Normed Spaces, Pergamon Press, Oxford, 1964.

[8] Potra, F. A., On Q-order and R-order of convergence, J. Optim. Theory Appl. 63(3) (1989), 415-431.

[9] Yamamoto, T., A convergence theorem for Newton-like methods in Banach space, Numer. Math. 51 (1987), 545-557.

[10] Ypma, T. J., Local convergence of inexact Newton methods, SIAM J. Numer. Anal. 21(3) (1984), 583-590.

[11] Zabrejko, P. P., Nguen, D. F., The majorant method in the theory of NewtonKantorovich approximations and the Ptak error estimates, Numer. Funct. Anal. Optim. 9 (1987), 671-684.

IOANNIS K. Argyros

Department of Mathematics

CAMERON University

LAWTON, OK 73505

USA

IOANNISA@CAMERON.EDU 